\title{
Propagación clonal in vitro de Paulownia elongata x Fortunei
}

In vitro clonal propagation of Paulownia elongata $x$ Fortunei

\author{
María Isabel Domínguez Rave ${ }^{1}$; Dagoberto Castro - Restrepo ${ }^{1}$ y Jesús Jaiber Díaz García ${ }^{1}$
}

DOI. 10.21931/RB/2019.04.01.5

\begin{abstract}
The hybrids of Paulownia elongata $X$ fortunei are considered as an interesting alternative for programs of production of biomass, raw material, the production of pulp and its use in processes of recovery of soils exploited by mining. Sexual propagation presents high genetic segregation, which is why the use of in vitro reproduction techniques is recommended. We used internodes and apices that after being disinfected with ethanol (70\%) and sodium hypochlorite (1\%) for ten minutes were established in the MS culture medium and different concentrations of BAP were evaluated $\left(0,0.5,1.0,1.5\right.$ and $\left.3.0 \mathrm{mgL}^{-1}\right)$ in combination with $\operatorname{AIB}(0,0.1$ and $1.0 \mathrm{mgL}-1)$ and for the rooting were evaluated concentrations of $\mathrm{AIB}\left(0.0 .5,1\right.$ and $\left.3.0 \mathrm{mgL}^{-1}\right)$. The best proliferation and shoot length results were obtained with concentrations of $1.5 \mathrm{mgL}-1$ of BAP with $0.1 \mathrm{mgL}-1$ of AIB with 4 shoots / explant and a length of $4 \mathrm{~cm}$. The rooting was achieved between $85 \%$ and $82 \%$ with the use of 1 and 3 mgL-1 of AIB respectively.
\end{abstract}

Keywords, micropropagation, in vitro proliferation, in vitro rooting.

Resumen: Los híbridos de Paulownia elongata X fortunei se consideran como una interesante alternativa para programas de producción de biomasa, materia prima la producción de pulpa y su utilización en procesos de recuperación de suelos explotados por minería. La propagación sexual presenta alta segregación genética por lo cual se recomienda la utilización de técnicas de reproducción in vitro. Se emplearon entrenudos y ápices los cuales después de ser desinfectados con etanol (70\%) e hipoclorito de sodio (1\%) durante diez minutos se establecieron en el medio de cultivo MS y se evaluaron diferentes concentraciones de BAP $\left(0,0.5,1.0,1.5\right.$ y $\left.3.0 \mathrm{mgL}^{-1}\right)$ en combinación con AIB $\left(0,0.1\right.$ y $\left.1.0 \mathrm{mgL}^{-1}\right)$ y para el enraizamiento se evaluaron concentraciones de AIB (0. 0.5, 1 y $\left.3.0 \mathrm{mgL}^{-1}\right)$. Los mejores resultados de proliferación y longitud de los brotes se obtuvieron con concentraciones de $1.5 \mathrm{mgL}^{-1}$ de BAP con $0.1 \mathrm{mgL}^{-1}$ de AIB con 4 brotes/explante y una longitud de $4 \mathrm{~cm}$. El enraizamiento se logró entre el $85 \%$ y $82 \%$ con la utilización de 1 y $3 \mathrm{mgL}^{-1}$ de AIB respectivamente.

Palabras clave: micropropagación, proliferación in vitro, enraizamiento in vitro.

\section{Introducción}

Paulownia es un género maderable de la familia Scrophulariaceae nativo de China y que ha sido adaptado en diversas partes del mundo tales como América y Europa. Se caracteriza por ser un árbol deciduo de rápido crecimiento, con grandes hojas distribuidas en pares opuestos en el tallo. Se utiliza para programas de reforestación en suelos pobres en nutrientes y debido a la amplia distribución de su sistema radical se puede emplear para la fitorremediación de suelos contaminados por actividades industriales ${ }^{1,2}$. La madera se utiliza para la producción de pulpa para la fabricación de papel y para enchapes. Paulownia tomentosa ha tenido mayor atención respecto a otras especies del género debido a que las primeras plantaciones se establecieron en Norteamérica. Sin embargo, investigaciones con $P$. elongata en América y Europa y $P$. fortunei en Australia han mostrado que sus características de crecimiento y manejo son mejores ${ }^{3}$. De igual manera, se han desarrollado híbridos de $P$. elongata $X$ fortunei que han mostrado ser promisorios.

La reproducción de $P$. elongata, $P$. fortunei y sus híbridos se puede realizar a partir de semilla; sin embargo, se presenta alta variabilidad genética y largos periodos de juvenilidad. Los métodos de propagación vegetativa son preferidos como es el caso de estacas que se enraízan con la utilización de reguladores de crecimiento, aunque la respuesta es inespecífica y no se producen altos porcentajes de enraizamiento ${ }^{4}$.

Por esta razón, la utilización de técnicas de reproducción in vitro de Paulownia sp han tomado especial importancia para suplir material para la industria forestal ${ }^{5,6}$. Las técnicas de propagación in vitro permiten la obtención de materiales libres de plagas y enfermedades, mayor homogeneidad fisiológica para la producción de biomasa y aprovechamiento forestal. Se tienen investigaciones de reproducción a partir de organogénesis directa e indirecta y mediante embriogénesis somática2,3,7,8,9.

La regeneración in vitro es influenciada por factores como el tipo de explantes, medios de cultivo, reguladores de crecimiento y condiciones ambientales ${ }^{10,11}$.

El objetivo del presente estudio fue el de establecer una metodología para la propagación clonal in vitro de un hibrido de Paulownia elongata X fortunei

\footnotetext{
${ }^{1}$ Grupo de Investigación Unidad de Biotecnologia, Universidad Católica de Oriente Rionegro, Colombia. orcid.org/0000-0003-3949-3263

${ }^{1}$ Grupo de Investigación Unidad de Biotecnologia, Universidad Católica de Oriente, Rionegro, Colombia. orcid. org/0000-0002-6599-9332

${ }^{1}$ Grupo de Investigación Unidad de Biotecnologia, Universidad Católica de Oriente, Rionegro, Colombia.
}

*Autor corresponsal: Universidad Católica de Oriente, investigacion y desarrollo, Sector 3, cra. 46 No. 40B 50, PBX: +(57) (4) 569 90 90, A.A. Rionegro: 008. Rionegro, Antioquia, Colombia. Email:dcastroinvestigacion@gmail.com. 


\section{Materiales y métodos}

\section{Área de estudio}

\section{Fuente de explantes}

Fueron empleadas yemas apicales y axilares de 2 centímetros de longitud, se realizó un lavado de estas yemas con yodo $(2 \mathrm{~mL} / \mathrm{L})$ durante 2 minutos. En cámara de flujo se desinfectaron con etanol 70\% durante 2 minutos en hipoclorito de sodio al $1 \%$ durante 10 minutos posteriormente, se realizó un triple lavado con agua destilada estéril. En el caso de las yemas axilares fueron tomados entrenudos con yemas de aprox. $0.3 \mathrm{~cm}$ de longitud, respecto a la yema apical se eliminaron los primordios foliares y se dejó una porción aproximada de 0.5 $\mathrm{cm}$. Se realizó el establecimiento en el medio de cultivo $\mathrm{MS}^{12}$ y se le adicionó las vitaminas MS, sacarosa (3\%) y agar - agar $\left(6.5 \mathrm{gL}^{-1}\right)$.

\section{Fase de proliferación}

Se evaluaron diferentes concentraciones de $\operatorname{BAP}(0,0.5$, 1.0, 1.5 y $3.0 \mathrm{mgL}^{-1}$ en combinación con $\operatorname{AIB}\left(0,0.1\right.$ y $\left.1 \mathrm{mgL}^{-1}\right)$. En la tabla 1 se muestran los tratamientos realizados. Se utilizó un experimento factorial $5 \times 3$ con diseño completamente al azar. Cada tratamiento estuvo compuesto por 5 repeticiones. Los datos se analizaron mediante ANOVA y se utilizó el análisis de contrastes de Tukey para determinar diferencia entre las medias de los tratamientos.

\begin{tabular}{|c|c|c|}
\hline Tratamientos & BAP $\left(\mathbf{m g L}^{-1}\right)$ & AIB $(\mathbf{m g L}-\mathbf{I})$ \\
\hline $\mathbf{1}$ & 0.0 & 0.0 \\
\hline $\mathbf{2}$ & 0.0 & 0.1 \\
\hline $\mathbf{3}$ & 0.0 & 1.0 \\
\hline $\mathbf{4}$ & 0.5 & 0.0 \\
\hline $\mathbf{5}$ & 0.5 & 0.1 \\
\hline $\mathbf{6}$ & 0.5 & 1.0 \\
\hline $\mathbf{7}$ & 1.0 & 0.0 \\
\hline $\mathbf{8}$ & 1.0 & 0.1 \\
\hline $\mathbf{9}$ & 1.0 & 1.0 \\
\hline $\mathbf{1 0}$ & 1.5 & 0.0 \\
\hline $\mathbf{1 1}$ & 1.5 & 0.1 \\
\hline $\mathbf{1 2}$ & 1.5 & 1.0 \\
\hline $\mathbf{1 3}$ & 3.0 & 0.0 \\
\hline $\mathbf{1 4}$ & 3.0 & 0.1 \\
\hline $\mathbf{1 5}$ & 3.0 & 1.0 \\
\hline
\end{tabular}

Tabla 1. Evaluación del efecto de la bencilaminopurina (BAP) y el ácido Indolbutírico (AIB) en la fase de proliferación in vitro de P. elongata $x$ P. fortunei.
Fase de enraizamiento. Se utilizó el medio MS y se evaluó el efecto del ácido indolbutírico (0, 0.5, 1 y $\left.3 \mathrm{mgL}^{-1}\right)$. Se utilizó un diseño completamente al azar con 30 repeticiones por tratamiento.

Para el análisis de la información se utilizó el paquete estadístico RWizard 3.1. En todos los casos se verificaron los supuestos de normalidad de Lilliefors (Kolmogorov-Smirnov), Shapiro-Wilk y de homogeneidad de varianza de Levene's.

\section{Resultados y discusión}

\section{Fase de proliferación}

De acuerdo con los análisis estadísticos se encontró interacción entre las concentraciones de BAP y AIB respecto al número de brotes / explante. En la figura 1 se observa como cuando se utilizó AIB (0.1 $\left.\mathrm{mgL}^{-1}\right)$ con las concentraciones de BAP hasta $1.5 \mathrm{mgL}^{-1}$ se incrementó el número de brotes, obteniendo su máximo valor en esta concentración. Con las concentraciones de AIB ( 0 y $1 \mathrm{mgL}^{-1}$ ) el máximo valor se alcanzó con BAP (1 mgL-1 $)$. En todos los casos concentraciones de BAP (3 $\mathrm{mgL}^{-1}$ ) causó disminución en el número de brotes.

Al realizar la comparación de las medias de los tratamientos (figura 2) se encontraron diferencias significativas entre estos respecto al número de brotes por explante. El tratamiento donde se utilizó BAP (1.5 mgL-1) más AIB (0.1 mgL-1) mostró un promedio de 4 brotes / explante con una periodicidad de subcultivos de cada 21 días. Estos resultados difieren con los obtenidos por ${ }^{4}$, con P. tomentosa con un promedio de 7.4 brotes por explante, lo cual se puede explicar por la especie que se evaluó. Es conocido el efecto fisiológico de las citoquininas en la estimulación de división y elongación celular y la activación de la síntesis de ARN, la estimulación de síntesis de proteínas y actividad enzimática como ha sido revisado por ${ }^{13}$. La combinación de BAP con AIB contribuye a mejorar la proliferación posiblemente debido a un equilibrio entre citoquininas / auxinas.

Respecto a la longitud de los brotes en la figura 3 se observó que en todos los casos la mayor longitud de los brotes se alcanzó con BAP (1.5 mgL-1), mientras que la concentración de BAP (1.5 $\left.\mathrm{mgL}^{-1}\right)$ disminuyó la longitud de los brotes. Al realizar la comparación de medias de tratamientos se encontró que cuando se utilizó BAP $\left(0.5,1.0,1.5\right.$ y $\left.3.0 \mathrm{mgL}^{-1}\right)$ con AIB (0.1 y $\left.1.0 \mathrm{mgL}^{-1}\right)$ se tuvo la mejor respuesta en cuanto a la longitud de los brotes (figura 4).

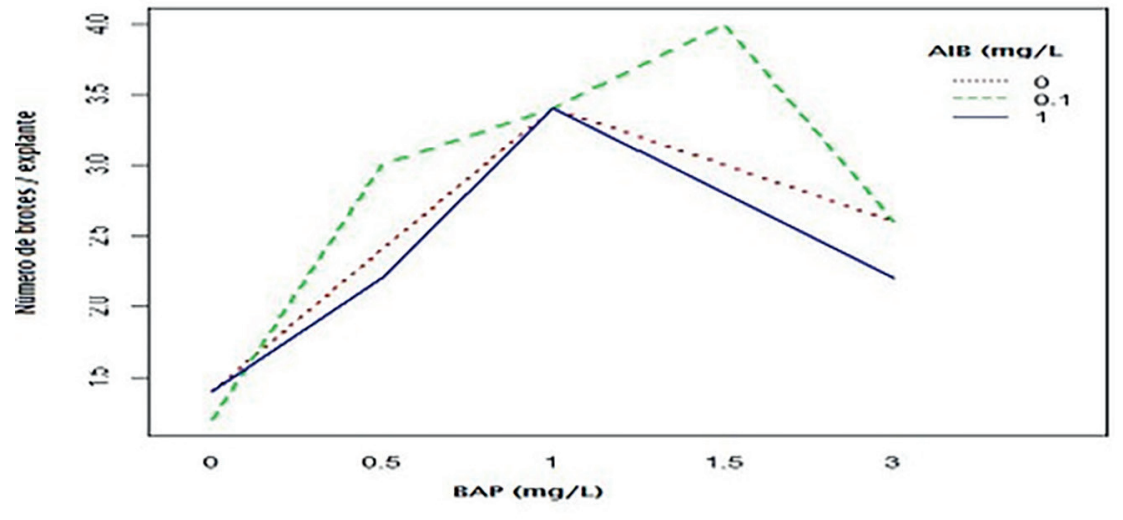

Figura 1. Interacción entre los factores correspondientes a diferentes concentraciones de BAP y AIB sobre el número de brotes in vitro en Paulownia elongata $X$ fortunei 
Figura 2. Evaluación del efecto del BAP y AIB sobre el número de brotes de P. elongata $\times$ P. fortunei
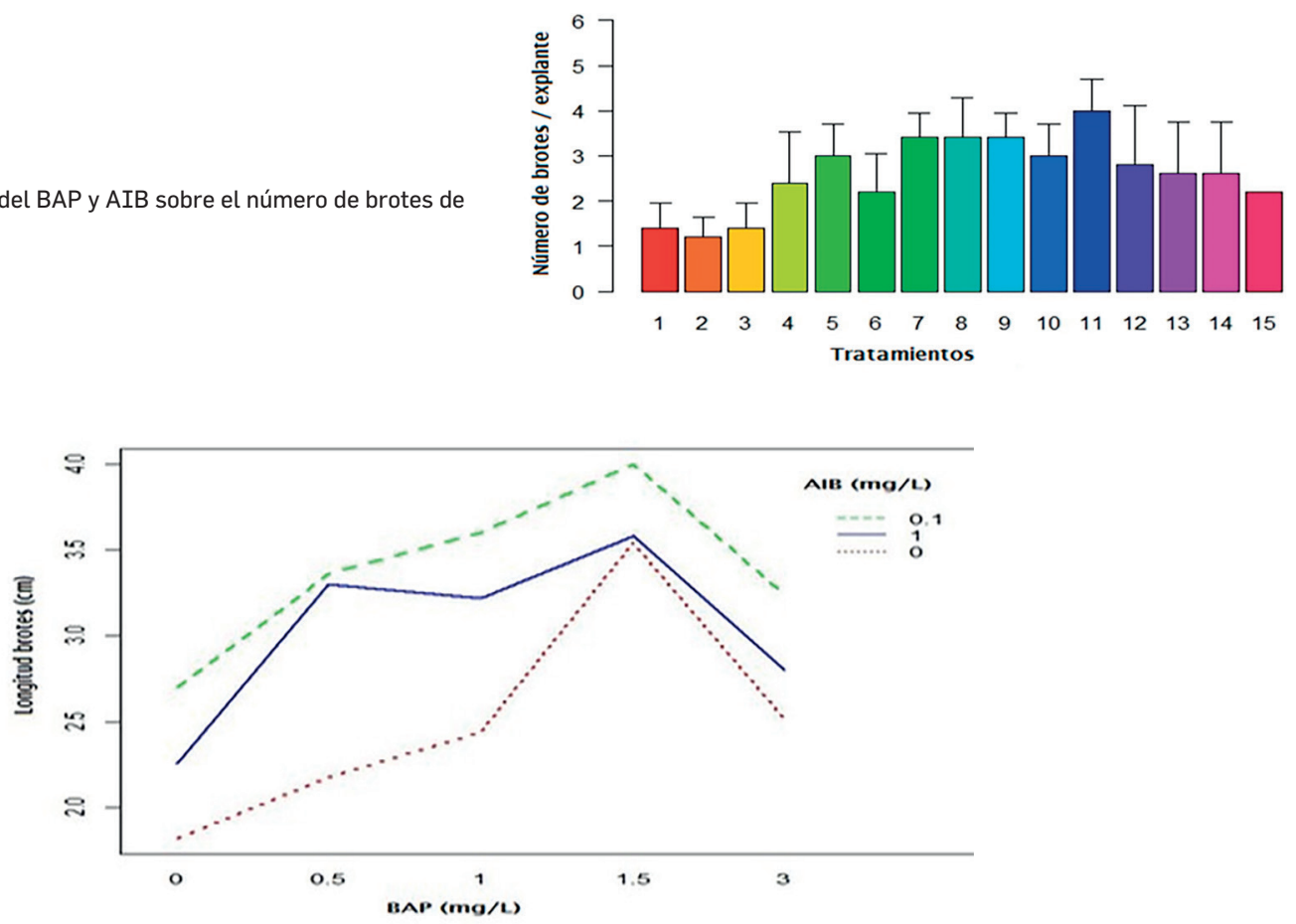

Figura 3. Interacción entre los factores correspondientes a diferentes concentraciones de BAP y AIB sobre la longitud de los brotes in vitro en Paulownia elongata $X$ fortunei

Figura 4. Evaluación del efecto del BAP y AIB sobre la longitud de los brotes de $P$. elongata $\times$ P. fortunei.

\section{Fase de enraizamiento}

Después de cuatro semanas se evaluó el efecto de cuatro concentraciones de AIB sobre el porcentaje de enraizamiento y número de raíces por explante. Respecto al porcentaje de enraizamiento (figura 5) las medias de los tratamientos presentaron diferencias significativas. De acuerdo con el test de Tukey se encontró que las concentraciones de 1 y $3 \mathrm{mgL}^{-1}$ presentaron los mayores porcentajes de enraizamiento del $85 \%$ y $82 \%$ respectivamente.

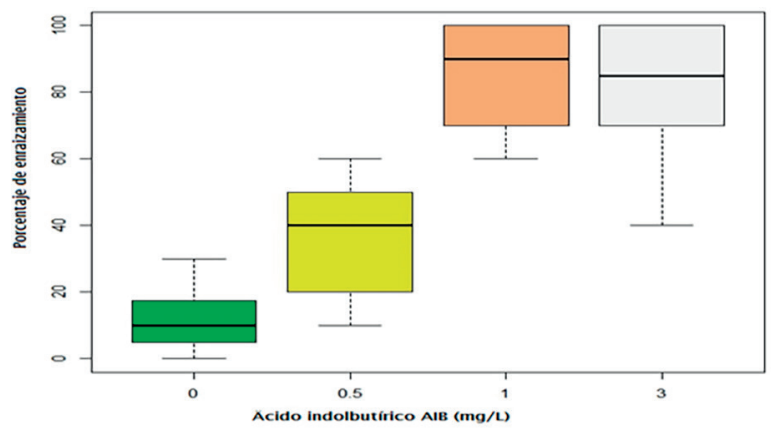

Figura 5. Efecto de diferentes concentraciones de AIB sobre el porcentaje de enraizamiento in vitro de brotes de $P$. elongata $x$ P. fortunei.

Con relación al número de raíces los tratamientos donde se utilizaron concentraciones de 1 y $3 \mathrm{mgL}^{-1}$ tuvieron la mejor respuesta con 3.4 y 3 raíces por brote respectivamente (figu-

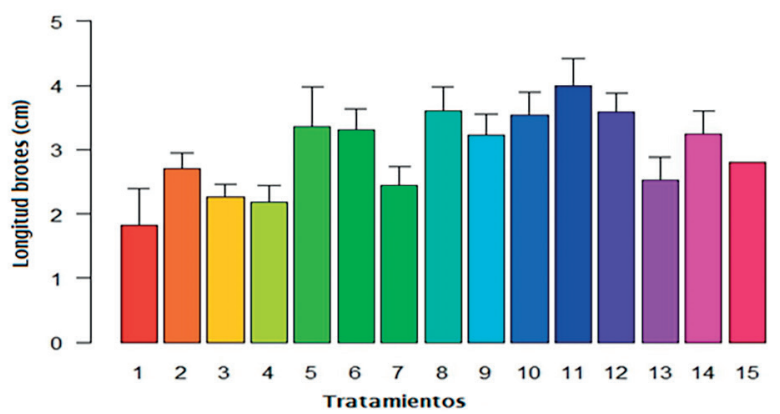

ra 6). Estos resultados coinciden con los encontrados por ${ }^{7}$. quienes encontraron porcentajes de enraizamiento del 66.6\% y 3.57 raíces/brote en $P$. kawakamii. De igual manera ${ }^{4}$ en $P$. tomentosa encontraron que el empleo de IBA $\left(0.5 \mathrm{mgL}^{-1}\right)$ tuvo la mejor respuesta de enraizamiento mayor del $95 \%$.

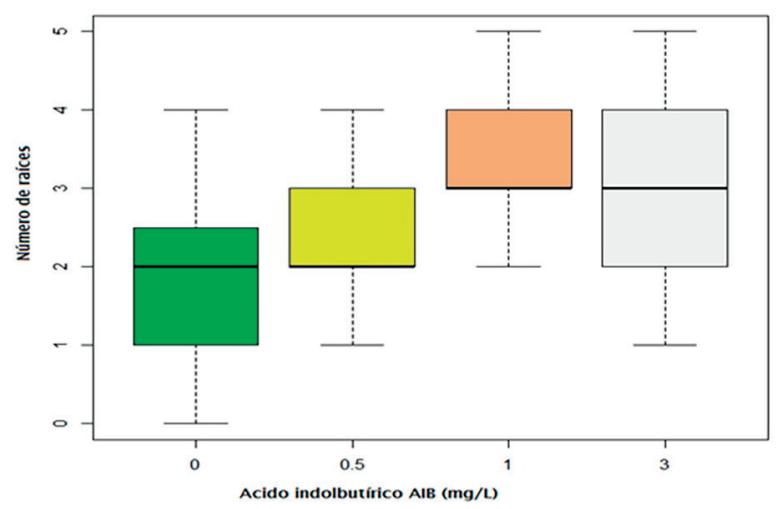

Figura 6. Efecto de diferentes concentraciones de AIB sobre el número de raíces in vitro de $P$. elongata $x P$. fortunei 
En la figura 7 se muestran los estados de desarrollo de $P$. elongata $\times P$. fortunei propagados in vitro. La primera fase de establecimiento se logró con la siembra de entrenudos los cuales se desinfectaron con etanol (70\%) durante dos minutos e hipoclorito de sodio (1\%) durante diez minutos. La proliferación se desarrolló en el medio de cultivo MS suplementado con BAP (1.5 mgL $\left.\mathrm{má}^{-1}\right)$ malB $\left(0.2 \mathrm{mgL}^{-1}\right)$, donde después de 60 días se estabilizó en la producción media de 4 brotes / explante cada cuatro semanas. El enraizamiento se desarrolló con AIB (1 $\left.\mathrm{mgL}^{-1}\right)$ con un porcentaje del $85 \%$ a los 30 días.

\section{Conclusiones y recomendaciones}

la tecnología de cultivo in vitro permite mejorar el acceso a una gran cantidad de plantas a partir de cantidades mínimas de material vegetal, y su desarrollo productivo representa una oportunidad de crecimiento y diversificación a nivel del sector forestal de nuestro país; asimismo, estos cultivos podrían usarse para predecir las respuestas de las plantas a los contaminantes ambientales, y así reducir el costo de los experimentos de plantas convencionales.

\section{Agradecimientos}

Los autores agradecen a la "Red para el aprovechamiento de los recursos naturales y obtención de productos biotecnológicos para suelos disturbados (RPBSD)", programa financiado por COLCIENCIAS en el convenio 576-2012, por la financiación al desarrollo de este trabajo.

\section{Referencias}

1. Zhu, Z. H., Chao, C. J., Lu, X. X., \& Xiong, Y. G. (1988). Paulownia in China cultivation and utilization. Asian Network for Biol. Sci. and Inter. Dev. Res. Centre, Singapore.

2. Castellanos Hernández, O. A., Rodríguez Sahagún, A., Rodríguez Domínguez, J. M., \& Rodríguez Garay, B. (2006). Organogénesis indirecta y enraizamiento in vitro de Paulownia elongata. e-Gnosis, (4).
3. Bergmann, B. A., \& Whetten, R. (1998). In vitro rooting and early greenhouse growth of micropropagated Paulownia elongata shoots. New forests, 15(2), 127-138.

4. Rahman, M. A., Rahman, F., \& Rahmatullah, M. (2013). In vitro regeneration of Paulownia tomentosa Steud. plants through the induction of adventitious shoots in explants derived from selected mature trees, by studying the effect of different plant growth regulators. American-Eurasian Journal of Sustainable Agriculture, 7(4), 259-268.

5. Angelova-Romova, M., Koleva, A., Antova, G., Zlatanov, M., Stoyanova, M., Dobreva, K., ... \& Stoyanova, A. (2011). Lipid composition of Paulownia seeds grown in Bulgaria. Trakya University Journal of Sciences, 13(2), 101-111.

6. BYLIANA IVANOVA, P., Yancheva, S., \& Bojinov, B. (2012). Molecular differentiation of Paulownia species and hybrids. Journal of Central European Agriculture, 13(1), 0-0.

7. Lobna, S. T., Ibrahim, M. S., \& Farahat, M. M. (2008). A Micropropagation Protocol of Paulownia kowakamii through in vitro culture technique. Australian Journal of basic and applied sciences, 2(3), 594-600.

8. Markovic, M., Vilotic, D., \& Popovic, M. (2013). Propagation of Paulownia elongata S. Y. Hu by axillary shoots. Propagation of Ornamental Plants, 13(2), 73-77.

9. Shtereva, L., Vassilevska-Ivanova, R., Karceva, T., \& Kraptchev, B. (2014). Micropropagation of six Paulownia genotypes through tissue culture. Journal of Central European Agriculture, 15(4), 0-0.

10. Giri, C. C., Shyamkumar, B., \& Anjaneyulu, C. (2004). Progress in tissue culture, genetic transformation and applications of biotechnology to trees: an overview. Trees, 18(2), 115-135.

11. Ozaslan, M., Can, C., \& Aytekin, T. (2005). Effect of explant source on in vitro propagation of Paulownia tomentosa Steud. Biotechnology \& Biotechnological Equipment, 19(3), 20-26.

12. Murrashige, T., \& Skoog, F. (1962). A revised medium for rapid growth and bioassays with tobacco tissue culture. Physiol. Plant, $15,473-497$.

13. Kulaeva, O. N. (1980). Cytokinin action on enzyme activities in plants. In Plant Growth Substances 1979 (pp. 119-128). Springer, Berlin, Heidelberg.

Recibido: 8 octubre 2018

Aprovado: 15 diciembre 2018

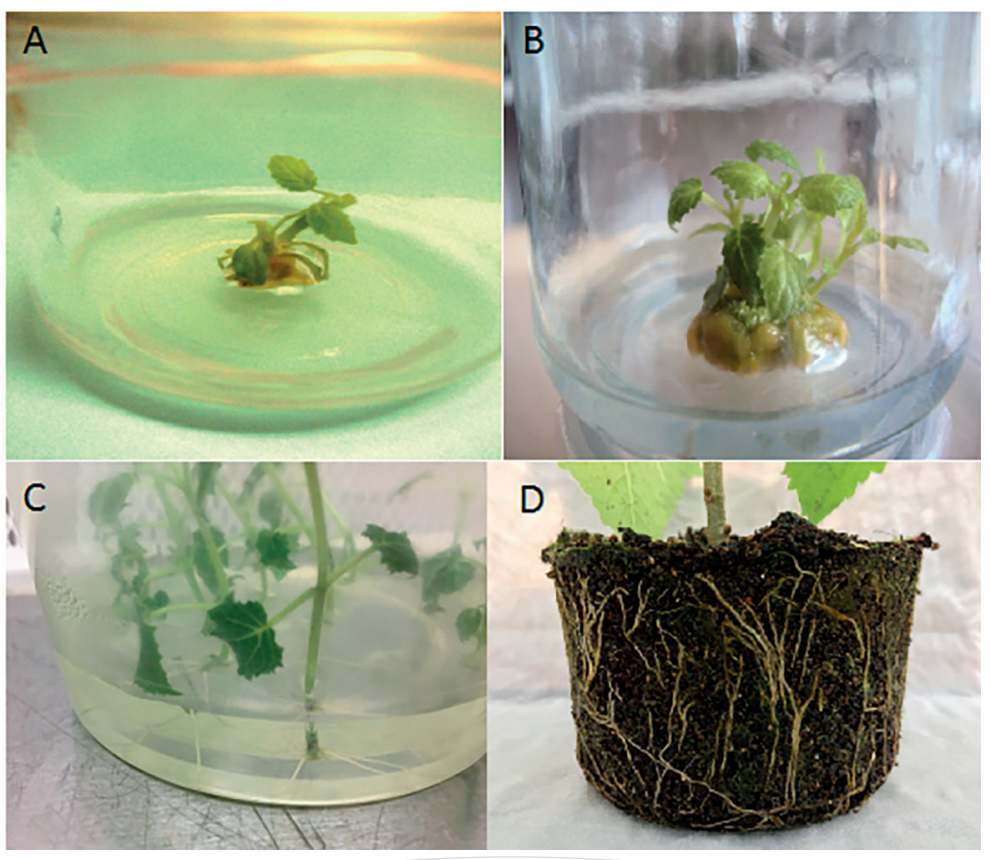

Figura 7. Diferentes fases de desarrollo de P. Paulownia elongata $X$ fortunei propagado in vitro. A. Fase de establecimiento. B. Fase de proliferación. C. Fase de enraizamiento. D. Aclimatización. 